\title{
Pediatric Anesthesia Techniques during the COVID-19 Pandemic
}

\author{
Alexander Trujillo Mejía ${ }^{1,2,3, \star}$, Fernando Arango ${ }^{1,2}$, Cristina Osorio ${ }^{3}$, \\ Federico Ocampo ${ }^{2,3}$, Lina Melo ${ }^{2,3}$, Leonardo Gil ${ }^{1,2,3}$
}

\author{
${ }^{1}$ Departmento Clínico Quirúrgico, \\ Universidad de Manizales, Manizales, \\ Colombia \\ ${ }^{2}$ Departmento Clínico Quirúrgico, \\ Universidad de Caldas, Manizales, \\ Colombia \\ ${ }^{3}$ Departmento de Anestesiología, \\ Hospital Infantil de la Cruz Roja, \\ Manizales, Colombia
}

\section{*Correspondence}

alexander.trujillo@ucaldas.edu.co (Alexander Trujillo Mejía)

\begin{abstract}
The pediatric population is a contagion fuel for SARS CoV-2, putting health care workers at risk. Exposure to respiratory droplets and aerosols are a major cause of disease transmission during airway procedures. Crying, coughing, laryngospasm, airway obstruction and hypoxia, which are more frequent in children than adults, directly or indirectly increase aerosol production and hence the risk of transmission to health workers. We present recommendations for the perioperative management of children suspected or confirmed positive for COVID-19, with the aim of reducing the risk of infection in these patients and in the healthcare team.
\end{abstract}

\section{Keywords}

COVID-19, Pediatric anesthesia, SARS CoV-2, Aerosol box, Airway

\section{What do we know about this topic?}

Adherence to recommendations and management guidelines when approaching pediatric patients during the COVID-19 pandemic reduces disease transmission.

\section{What does the study contribute?}

This study provides recommendations on pediatric anesthesia techniques that decrease aerosol production and improve the safety of patients and healthcare personnel during the COVID19 pandemic.

\section{Introduction}

The SARS-CoV-2 pandemic affects all ages [1]. Children have a lower incidence $(0.9 \%-1.2 \%)$ and a moderate clinical picture [2]. In Wuhan, the attack rate was $12.3 \%$ in children and it has been estimated the pediatric population represents between 1.2 and $2 \%$ of all infected cases [3]. In Spain, $60 \%$ of infected children required hospitalization, of which $9.7 \%$ was in the Intensive Care Unit. In Colombia, 2 deaths have been reported in pediatric patients with cardiovascular disease, although most have a good prognosis $[4,5]$.

At the beginning of the illness, infected children have fever, cough, headache, runny nose, vomiting and diarrhea. One week after initiation, they may develop symptoms of systemic toxicity such as general malaise, asthenia and decreased oral intake. The clinical condition in some children may rapidly worsen and cause respiratory failure. These severe cases can end in septic shock, metabolic acidosis and coagulation disorders. However, most children recover within 1 to 2 weeks $[4,5]$. The leukocyte value is generally normal and lymphopenia is not evident in most cases, suggesting a lower immune response in SARS CoV-2 infection [5].

The pediatric population has a similar probability of viral transmission as adults [6], even if they are asymptomatic [5] and must undergo intense preventive measures [6]. Therefore, the healthcare team should adopt a high index of suspicion, especially during the airway approach. This is because children can be contagious even in the absence of symptoms.

China and Spain reported more than 3,300 and 12,298 infected healthcare workers, respectively, as of March 2020 $[7,8]$. The United Kingdom reported 4,605 cases in mid-April [7], and 678 cases were reported in Colombia on May 9 [9].

Crying, coughing, laryngospasm, airway obstruction and hypoxia are more frequent in children than adults and can directly or indirectly increase aerosol production and hence the risk of transmission to health professionals. This article will review pediatric anesthesia techniques during the COVID-19 pandemic with the aim of avoiding the above situations and thus improving the safety of patients and health workers.

\section{Transmission of the virus}

Spread of the SARS CoV-2 virus occurs through respiratory droplets and contact routes [10]. The respiratory droplets are larger than $5 \mu \mathrm{m}$ in diameter and subject to gravitational force, so their dispersion reaches up to one or two meters away. Transmission by contact occurs because the virus remains on surfaces for hours to days, thereby being a potential source of infection [11].

Air transport occurs when particles smaller than $5 \mu \mathrm{m}$ in diameter (aerosols) circulate in the air for long periods of time and for distances of greater than 2 meters [12]. The virions in these particles are absorbed by the respiratory mucosa and even by the conjunctiva. Procedures involving the airway can gener- 
TA B L E 1. Recommendations for safe anesthetic technique for children with COVID-19.

\section{Before the procedure}

Sanitation of hands.

Adequate use of personal protection elements.

If possible, perform the procedure in a room with more than 12 air changes per hour and with negative pressure.

Reduce the number of people in the room.

Enlist aerosol box.

Have all the devices for airway management checked; package and label all medications.

Have HEPA filters in place (risk of hypercapnia in young children).

salbutamol in bronchial hyper-reactivity or recent respiratory symptoms.

Premedication: oral or venous anxiolytic, atropine to decrease oral secretions (caution), Venous cannulation before entering the operating room.

Use of cognitive aids.

\section{During the procedure}

Put aerosol box on the patient's head, cover the distal hole of the box on the patient's abdomen.

Conventional monitoring.

Pre-oxygenation: $\mathrm{FiO}_{2} 80 \%$. Achieve exhaled $\mathrm{O}_{2}$ concentration of 70 Intravenous induction of fast or fast modified sequence.

Avoid giving positive pressure ventilation through a face mask.

Video laryngoscope or conventional laryngoscope according to expertise.

Fix the OTT very well to the patient and to the anesthetic circuit.

Protective mechanical ventilation: $6 \mathrm{ml} / \mathrm{Kg}$, RR 35/min or less according to age, plateau pressure less than $30 \mathrm{cmH}_{2} \mathrm{O}$, moderately elevated levels of PEEP $\left(5 \mathrm{~cm} \mathrm{H}_{2} \mathrm{O}\right)$ and up should be titrated.

Recruitment with IS pressure of $30-40 \mathrm{~cm} \mathrm{H}_{2} \mathrm{O}$ for 15 - 20 seconds.

Using cognitive aids.

\section{After the procedure}

Avoid disconnections from the circuit.

Extubation:

Avoid cough and laryngospasm.

Preferably do it with the deep patient, with spontaneous ventilation and in lateral decubitus.

Administer venous lidocaine 3 minutes before. Consider dexmedetomidine.

Have a proper extubation MAC.

Guarantee good $\mathrm{SpO}_{2}$ and adequate respiratory effort.

Inside the aerosol box.

Immediately put on the surgical mask after extubation.

Recover in surgery room.

Caution when removing personal protection elements.

Use cognitive aids.

Abbreviations: HEPA: High Efficiency Particulate Air; $\mathrm{FiO}_{2}$ : Inspirated fraction of oxygen. OTT: Orotracheal Tube; RR:

Respiratory Rate; PEEP: Positive Pressure at End of Expiration; $\mathrm{SpO}_{2}$ : Pulse Oximetry. Source: Authors.

ate aerosols that carry the virus across distances. These include tracheal intubation and extubation, tracheostomies, cricothyroidotomies, positive pressure ventilation (PPV), open tracheal aspiration, bronchoscopy, bronchoalveolar lavage, sputum induction and modes of non-invasive ventilation [13]. The WHO recommends drop and contact precautions on a routine basis and aerosol precautions for these procedures [14].

\section{Air exchange in the room}

Whenever possible, airway manipulation should be performed in a negative pressure room with the doors closed. However, rapid renewal of air in the room (more than 12 times per hour) is more important than pressurization of the room [13]. 


\section{Personal protection equipment}

The personal protection elements (PPE) necessary to carry out these procedures consist of: gloves, long-sleeved anti-fluid gown, eye protection that can be glasses or a mask (personal glasses are insufficient), FFP3 mask and gloves [10] (Table 1). It is highly recommended to use checklists for setting and removing these elements. In addition, the principles of infection prevention and control must be followed.

\section{Mechanical barriers against aerosols}

The WHO recommends using mechanical barriers against aerosols. The aerosol box has been rapidly introduced as an effective barrier method. The part that covers the patient's abdomen allows aerosols to escape and therefore it is recommended that it be covered. Other forms of protection involving plastic covers with controlled access have also been suggested [15].

\section{Preparation}

Before taking the patient to the surgery room, different devices of all sizes should be prepared for airway management according to age. Packaged and labeled medications must be available. Also recommended are an intubation guide, protected Kelly forceps, and a cap for the end of the tracheal tube. The suction system must be closed to avoid production of aerosols.

\section{Use of filters in the anesthetic circuit}

The HEPA (High Efficiency Particulate Air) antiviral filters placed in the anesthetic circuit protect those who manipulate the airway. Although its use is not a problem in adults and older children, in neonates and young infants the dead space produced by the filter can cause significant hypoventilation with severe hypercapnia, as has already been reported [16]. The filter must be chosen according to its dead space and the required tidal volume range (VC). A filter with a $10 \mathrm{~mL}$ dead space and a VC range between 30 and $200 \mathrm{~mL}$ may be too large for a $1000 \mathrm{~g}$ premature infant, but too small for a $30 \mathrm{~kg}$ patient with a $240 \mathrm{~mL} \mathrm{VC}$. If the filter is too big for the size of the infant it can lead to hypercapnia and intraventricular hemorrhage. Alternatively, a small filter used for a larger infant can cause increased airway resistance [17]. Therefore, it is important to always monitor the exhaled $\mathrm{CO}_{2}$ and airway pressures during use.

\section{Premedication}

About $70 \%$ of children experience significant stress and anxiety prior to surgery [18]. Negative anticipation before the procedure can generate psychomotor agitation, crying, screaming and other behaviors that force healthcare personnel to limit the patient's movements, thereby exposing themselves to droplets and respiratory aerosols. Adequate preoperative anxiolysis can therefore decrease the risk of contagion in health care workers. Premedication of the child via oral or intravenous routes (if available) before transfer to the operating room is recommended. Midazolam or clonidine are effective as preoperative anxiolytics in children [18]. The nasal route should be avoided because it can cause coughing or sneezing and the generation of aerosols [19]. Parental presence during anesthetic induction is not recommended due to the risk of contagion for parents and health care workers, as well as the need for additional PPEs that are scarce due to the pandemic [19]. Atropine is useful in preventing bradycardia and decreasing oral and respiratory secretions before anesthetic induction. However, it can thicken the secretions and predispose to atelectasis. In patients with upper airway infection or a history of bronchial hyper-reactivity, salbutamol premedication with a spacer chamber is useful to prevent aerosol formation [20].

\section{Venous cannulation}

It is important to have patent venous access before entering the operating room. Intravenous induction is faster and reduces the time of exposure to respiratory droplets and aerosols generated during the tracheal intubation process [21]. However, attempts at venous cannulation can trigger drop-generating behaviors in the child. There are some alternatives that, alone or in combination, improve the safety of the procedure. The "gold standar" is the application of topical anesthetic (EMLA) from 30 to 60 minutes before puncture. Videos, games and devices that generate vibration and cold (BUZZY) are also useful $[22,23]$.

\section{Pre-oxygenation}

Children, especially neonates and infants, are prone to hypoxemia during airway procedures, even in the absence of cardiorespiratory pathologies. Bradycardia and secondary circulatory collapse are more likely if respiratory infections are ongoing. Despite adequate pre-oxygenation, their high oxygen consumption and low residual lung capacity predisposes children to alveolar collapse. To prevent hypoxemia, the use of high-flow nasal cannulas that provide apneic oxygenation during intubation has been suggested. These cannulas have been useful in adults, but their utility in the pediatric population is disputed for several reasons. First, they have not been shown to perform better than conventional nasal cannulas. Second, the oxygen flow per kilogram of weight to use in order to avoid severe hypercapnia has not been determined. Third, they have a high cost and greater space and time requirements. Furthermore, due to the production of aerosols [21], highflow nasal cannulas can only be used in negative pressure environments. Therefore, it is reasonable to perform preoxygenation with $\mathrm{FiO}_{2}$ (inspired oxygen fraction) greater than $80 \%$ for two or three minutes before intubation (or until the exhaled fraction of oxygen is greater than $70 \%$ on the gas analyzer). Use two sources of oxygen: the anesthetic face mask and the classic nasal cannula with an oxygen flow of between 2 and 4 liters per minute. In addition to the PPE, use a barrier method (eg. the aerosol box) [24]. 


\section{Anesthetic induction and tracheal intubation}

Intravenous induction is preferred over inhalation. PPV should be avoided, which is why it is very important to do adequate pre-oxygenation. Some patients, especially the smallest or those with respiratory pathology, will require PPV to avoid hypoxemia. In these cases it should be administered by two people: one to adjust the face mask and the other for ventilation. The pressure exerted must be the minimum necessary to produce expansion of the thorax. It is recommended to minimize the flow of fresh gas to decrease the production of aerosols.

The video laryngoscope, preferably with a screen separate from the handpiece, can reduce the operators' exposure to secretions and aerosols from the airway [19, 25]. The success rate with this device depends on the training and experience of the operator. Despite achieving an adequate visualization of the structures, the insertion of the endotracheal tube is not easy, thus increasing the frequency of hypoxemia [25]. These factors can oblige giving PPV, thus increasing the exposure time to droplets and aerosols, which is to be avoided. For these reasons, the video laryngoscope is the best alternative if trained people are available, otherwise the conventional laryngoscope may be less risky for both patients and the operators.

Special attention should be paid to the fixation of the tracheal tube to prevent its displacement, extubation or disconnection, requiring additional manipulation of the airway. Cuffed tubes should be used.

\section{Mechanical ventilation and lung protection}

The protective ventilation guidelines extrapolated from the clinical guidelines for Acute Respiratory Distress Syndrome in adults emphasize four aspects: (1) CV less than or equal to $6 \mathrm{~mL} / \mathrm{kg}$ of ideal weight; (2) Respiratory rate 35 breaths $/ \mathrm{min}$ or less according to age; (3) Plateau pressure in the airway up to $30 \mathrm{~cm} \mathrm{H}_{2} \mathrm{O}$; (4) Positive pressure at the end of expiration (PEEP) from $5 \mathrm{~cm} \mathrm{H} \mathrm{H}_{2} \mathrm{O}$ and up [26, 27]. The tidal volume can start at $8 \mathrm{~mL} / \mathrm{kg}$ and decrease or remain there as long as it does not exceed the target of the mentioned plateau pressure $[27,28]$. In addition, it is advisable to maintain a conduction pressure (plateau pressure-PEEP) below 12 to $15 \mathrm{~cm} \mathrm{H}_{2} \mathrm{O}$ through adjustments in tidal volume and PEEP [29].

Evidence for the benefit of protective ventilation in pediatrics is scant. In general, studies are limited to newborns requiring mechanical ventilation in the ICU due to respiratory distress syndrome [30]. In a study of lung resection surgery with separate, one lung ventilation in children under 6 years of age, protective ventilation was found to reduce postoperative lung complications compared to conventional ventilation [30]. Current consensus during the COVID-19 pandemic recommends the strategy of moderate $\mathrm{VC}(7-8 \mathrm{~mL} / \mathrm{kg})$. This can use short cuffed tubes to avoid the dispersion of aerosols and to avoid the risk of ventilating only the dead space $[1,28]$.

Lung recruitment can be achieved through sustained inflation (SI) or by gradually increasing PEEP. The safety and efficacy of SI in pediatrics have been determined when main- taining a pressure of $30-40 \mathrm{~cm} \mathrm{H}_{2} \mathrm{O}$ for 15 - 20 seconds. Qiu and colleagues advocate a method to determine a specific SI figure based on 5 times the Mean Airway Pressure (MAP). This method provides optimal recruitment measured by oxygenation, lung dynamics, hemodynamic stability and lung histology [31].

Based on the Wuhan experience, adult patients with acute hypoxemic respiratory failure due to COVID-19 have poor tolerance to high PEEP, probably as a direct result of severe lung damage from the virus and inflammatory reaction. PEEP was successful in this patient population of less than $10 \mathrm{~cm}$ $\mathrm{H}_{2} \mathrm{O}$. As pulmonary recruitment maneuvers, PEEP should kept under $20 \mathrm{~cm} \mathrm{H}_{2} \mathrm{O}$ and decreased in increments of 2 to $3 \mathrm{~cm} \mathrm{H}_{2} \mathrm{O}$ as long as the oxygenation, plateau pressure and compliance objectives are maintained [28].

\section{Extubation}

Coughing-is one of the main sources of human-to-human contact for respiratory viral diseases [32] and should be considered a high-risk complication for health personnel due to the production of droplets and respiratory aerosols [33]. Laryngospasm is more frequent in children than in adults and may require PPV, thus increasing the risk of exposure to droplets and aerosols.

Unlike anesthetic induction where the anesthesiologist may have some control over the situation, extubation and anesthetic emergence have a higher degree of uncertainty and hence an extubation protocol should be followed. Several authors have found that extubation with the anesthetized patient reduces the risk of cough and desaturation, but increases the risk of airway obstruction compared to awake patient extubation [34]. Furthermore, the patient's position during extubation is also associated with different outcomes. With the patient deep and in lateral decubitus, the best oxygen saturation values $\left(\mathrm{SpO}_{2}\right)$ are obtained in the following 5 minutes compared to extubation in the supine position [35].

To achieve deep extubation with the patient free of cough and laryngospasm, it is important to keep in mind the exhaled value of the halogenated gases. For this purpose, an effective dose (ED) of $95 \%$ in children has been defined for extubation with sevoflurane (MAC $\mathrm{MXT95}_{\text {) }}$ of 1.9\% [36] and disfluorane $8.5 \%$ [37].

There is evidence for the effectiveness of some medications in preventing cough during extubation. In adults, dexmedetomidine, remifentanil, fentanyl and lidocaine are better than placebo [38]. In children, dexmedetomidine can facilitate a smooth extubation process and reduce the requirement for sevoflurane and emergency agitation when administrated perioperatively with minimal respiratory depression. A reduction in the frequency of laryngospasm and cough of $29.90 \%$ and $18.92 \%$, respectively, was reported in children when using 1.5 $\mathrm{mg} / \mathrm{kg}$ intravenous lidocaine for 3 minutes before extubation compared to placebo [39].

Before extubation, adequate $\mathrm{SpO}_{2}$ and respiratory effort must be guaranteed. Gastric ultrasound can be useful to decrease the possibility of deep extubation in patients with suspected full stomach.

In accordance with the above, it is reasonable to perform 
extubation with the patient deep in spontaneous ventilation, with adequate respiratory effort and lying on their side opposite the location of the assistant for extubation.

During the procedure, the following recommendations may be helpful: aspirate the oropharyngeal secretions with a closed system, put on nasal glasses of adequate size for the patient's age, close the flow of fresh gases just before the procedure, and remove the connected trachea tube to the filter and circuit. The use of a clamp on the orotracheal tube during extubation with the patient under spontaneous ventilation can produce negative pressure pulmonary edema and is not recommended.

\section{Anesthetic recovery}

Patients must recover within the surgery room. Specific attention should be paid to the patency of the airway and $\mathrm{SpO}_{2}$. The patient should wear a surgical mask and, if possible, remain inside the aerosol box. Post-operative pain, hypothermia, vomiting and agitation should be avoided through multimodal strategies, as these can be risk factors for healthcare personnel.

\section{Conclusion}

The pediatric population can be an important source of viral transmission, even in the absence of symptoms. During the perioperative care of pediatric patients, laryngospasm, airway obstruction, desaturation requiring positive pressure ventilation, psychomotor agitation, cough, tracheal intubation, and extubation represent high risk situations for healthcare personnel. During the airway approach, health workers must all wear PPE. A marked decrease in contagion both in healthcare personnel and in patients and their families can be achieved through the use of filters in the anesthetic circuits, the aerosol box, as well as the implementation of a methodical and orderly protocol during the patient's transit from entry into the institution until their anesthetic recovery and discharge.

\section{ACKNOWLEDGEMENTS}

Thanks to all the peer reviewers and editors for their opinions and suggestions.

\section{CONFLICTS OF INTEREST}

We declare that we do not have any commercial or associative interest that represents a conflict of interest in connection with the work submitted.

\section{REFERENCES}

[1] Marraro GA, Spada C. Consideration of the respiratory support strategy of severe acute respiratory failure caused by SARS-CoV-2 infection in children. Zhongguo Dang Dai Er Ke Za Zhi. 2020;22:183-194.

[2] Calvo C, García López-Hortelano M, de Carlos Vicente JC, et al. Recommendations on the clinical management of the COVID-19 infection by the «new coronavirus» SARS-CoV2. Spanish Paediatric Association working group. An Pediatr (Barc). 2020;92:241.e1-241.e11.

[3] Lee-Archer P, Ungern-Sternberg BS. Pediatric anesthetic implications of COVID-19-A review of current literature. Pediatr Anesth. 2020.

[4] Chen ZM, Fu JF, Shu Q, et al. Diagnosis and treatment recommendations for pediatric respiratory infection caused by the 2019 novel coronavirus. World J Pediatr. 2020;16:240-246.

[5] Cao Q, Chen Y-C, Chen C-L, et al. SARS-CoV-2 infection in children: Transmission dynamics and clinical characteristics. J Formos Med Assoc. 2020;119:670-673.

[6] Castagnoli R, Votto M, Licari A, et al. Severe Acute Respiratory Syndrome Coronavirus 2 (SARS-CoV-2) Infection in Children and Adolescents: A Systematic Review. JAMA Pediatr. 2020.

[7] Carl Heneghan, Jason Oke, Tom Jefferson. COVID-19 How many Healthcare workers are infected? CEBM. 2020. Available from: https://www. cebm.net/covid-19/covid-19-how-manyhealthcare-workers-are-infected/.

[8] Ludvigsson JF. Systematic review of COVID-19 in children shows milder cases and a better prognosis than adults. Acta Paediatr. 2020;109:10881095.

[9] COVID-19 en personal de salud en Colombia, Boletín No.6. Instituto Nacional de Salud de Colombia. 2020. Available in: https://www.ins.gov. co/COVID19PERSONALDESALUDCOLOMBIA/ boletin-6-covid-personal-salud.jpg.

[10] Public Health England. COVID-19: infection prevention and control guidance. Available from: https://www.gov.uk/government/ publications/wuhan-novel-coronavirus-infectionprevention-and-control/covid-19-personal-protectiveequipment-ppe.

[11] Van Doremalen N, Bushmaker T, Morris DH, et al. Aerosol and Surface Stability of SARS-CoV-2 as Compared with SARS-CoV-1. N Engl J Med. 2020;382:1564-1567.

[12] Wen X, Li Y. Anesthesia Procedure of Emergency Operation for Patients with Suspected or Confirmed COVID-19. Surg Infect (Larchmt). 2020;21:299-299.

[13] Cook TM. Personal protective equipment during the COVID-19 pandemic - a narrative review. Anaesthesia. 2020;75:920-927.

[14] World Health Organization. Modes of transmission of virus causing COVID-19: implications for IPC precaution recommendations. 2020. Available from: https://ww.who.int/news-room/ commentaries/detail/modes-of-transmission-of-viruscausing-covid-19-implications-for-ipc-precautionrecommendations.

[15] Yang Y-L, Huang C-H, Luk H-N, et al. Adaptation to the Plastic Barrier Sheet to Facilitate Intubation during the COVID-19 Pandemic. Anesth Analg. 2020.

[16] Schrock CR, Montana MC. Rapid COVID-19-related Clinical Adaptations and Unanticipated Risks. Anesthesiology. 2020;133:248.

[17] Trujillo A, Jaramillo X. Considerations for the Use of Respiratory Filters in Children During the COVID-19 Pandemic. Surg Infect (Larchmt). 2020.

[18] Rosenbaum A, Kain ZN, Laisson P, et al. The place of premedication in pediatric practice. Pediatr Anesth. 2009;19:817-828.

[19] Consenso recomendaciones anestesia pediatrica sección anestesia pediatrica. SEDAR. 2020. Available in: https://www.sedar.es/ images/site/NOTICIAS/coronavirus/2020_Ped_Covid19_ recomendaciones_final.pdf.

[20] Von Ungern-Sternberg BS, Salbutamol premedication in children with a recent respiratory tract infection. Paediatr Anaesth. 2009;19:1064-1069.

[21] Wax RS, Christian MD. Practical recommendations for critical care and anesthesiology teams caring for novel coronavirus (2019-nCoV) patients. Can J Anesth. 2020;67:568-576.

[22] Ballard A, Khadra C, Adler S, et al. External cold and vibration for pain management of children undergoing needle- related procedures in the emergency department: a randomised controlled non-inferiority trial protocol. BMJ Open. 2019;9:e023214.

[23] Ballard A, Khadra C, Adler S, et al. Efficacy of the Buzzy Device for Pain Management During Needle-related Procedures. Clin J Pain. 2019;35:532-543.

[24] Else SDN, Kovatsis PG. A Narrative Review of Oxygenation During Pediatric Intubation and Airway Procedures. Anesth Analg. 2020;130:831840 .

[25] Abdelgadir IS, Phillips RS, Singh D, et al. Videolaryngoscopy versus direct laryngoscopy for tracheal intubation in children (excluding neonates). Cochrane Database Syst Rev. 2017;5:CD011413. 
[26] Petrucci N, De Feo C. Lung protective ventilation strategy for the acute respiratory distress syndrome. Cochrane Database Syst Rev. 2013;2013:CD003844.

[27] Acute Respiratory Distress Syndrome Network, Brower RG, Matthay MA, et al. Ventilation with lower tidal volumes as compared with traditional tidal volumes for acute lung injury and the acute respiratory distress syndrome. N Engl J Med. 2000;342:1301-1308.

[28] Meng L, Qiu H, Wan L, et al. Intubation and Ventilation amid the COVID19 Outbreak: Wuhan's Experience. Anesthesiology. 2020;132; 13171332.

[29] Amato MBP, Meade MO, Slutsky AS, et al. Driving Pressure and Survival in the Acute Respiratory Distress Syndrome. N Engl J Med. 2015;372:747-755.

[30] Lee JH, Bae J il, Jang YE, et al. Lung protective ventilation during pulmonary resection in children: a prospective, single-centre, randomised controlled trial. Br J Anaesth. 2019;122:692-701.

[31] Jauncey-Cooke J, East CE, Bogossian F. Paediatric lung recruitment: A review of the clinical evidence. Paediatr Respir Rev. 2015;16:127-132.

[32] Aminnejad R, Salimi A, Saeidi M. Lidocaine during intubation and extubation in patients with coronavirus disease (COVID-19). Can J Anesth. 2020;67;759.

[33] Trujillo A. Response to Wen and Li, Anesthesia Procedure of Emergency Operation for Patients with Suspected or Confirmed COVID-19. Surg Infect (Larchmt). 2020;21:398.

[34] Koo C-H, Lee S, Chung S, et al. Deep vs. Awake Extubation and
LMA Removal in Terms of Airway Complications in Pediatric Patients Undergoing Anesthesia: A Systemic Review and Meta-Analysis. J Clin Med. 2018;7:353.

[35] Jung H, Kim HJ, Lee Y-C, et al. Comparison of lateral and supine positions for tracheal extubation in children. Anaesthesist. 2019;68:303308.

[36] Inomata S, Suwa T, Toyooka H, et al. End-Tidal Sevoflurane Concentration for Tracheal Extubation and Skin Incision in Children. Anesth Analg. 1998;87:1263-12677.

[37] Cranfield KAW, Bromley LM. Minimum alveolar concentration of desflurane for tracheal extubation in deeply anaesthetized, unpremedicated children. Br J Anaesth. 1997;78:370-371.

[38] Tung A, Fergusson NA, Ng N, et al. Medications to reduce emergence coughing after general anaesthesia with tracheal intubation: a systematic review and network meta-analysis. Br J Anaesth. 2020;124:480-495.

[39] Bhat S, Sanikop C. Efficacy of intravenous lidocaine in prevention of post extubation laryngospasm in children undergoing cleft palate surgeries. Indian J Anaesth. 2010;54:132.

How to cite this article:Alexander Trujillo Mejía, Fernando Arango, Cristina Osorio, Federico Ocampo, Lina Melo, Leonardo Gil. Pediatric Anesthesia Techniques during the COVID-19 Pandemic. Signa Vitae. 2020;16(2):8-13. doi:10.22514/sv.2020.16.0045. 